\title{
Prolonged antimicrobial activity of PMMA bone cement with embedded gentamicin releasing silica nanocarriers
}

\author{
by \\ Stefano Perni ${ }^{1}$, Sergio Caserta ${ }^{2}$, Rossana Pasquino ${ }^{2}$, Steve A. Jones ${ }^{3}$, Polina \\ Prokopovich ${ }^{1, *}$
}

${ }^{1}$ School of Pharmacy and Pharmaceutical Sciences, Cardiff University, Cardiff, UK

${ }^{2}$ Department of Chemical, Materials and Industrial Production Engineering, University of Naples Federico II, Italy

${ }^{3}$ University Hospital Llandough, Cardiff \& Vale University Health Board, Penlan Rd, Penarth CF64 2XX, Vale of Glamorgan, Wales, UK.

* Corresponding author:
Dr. Polina Prokopovich $\quad$ e-mail: prokopovichp@cardiff.ac.uk

School of Pharmacy and Pharmaceutical Sciences

Cardiff University

Redwood Building, King Edward VII Avenue

Cardiff, UK

CF10 3NB

Tel: $+44(0) 2920875820$

Fax: +44 (0)2920874149

Keywords: silica, bone cement, PBAE, antimicrobial, gentamicin 


\section{Abstract}

Antibiotic laden bone cements are regularly employed to prevent infections after joint replacement surgeries. We have developed silica nanocarriers loaded with gentamicin as a drug delivery systems to be dispersed in Poly-methyl-methacrylate (PMMA) bone cement for controlling and extending the release of the antibiotic from bone cements, thus proving a prolonged antimicrobial activity,. Layerby-Layer self-assembly was used to deposit gentamicin between alginate layers and two different Poly-beta-amino esters on the silica nanoparticles.

The release of gentamicin from PMMA bone cement containing silica nanocarriers continued for about 30 days compared to 6 days when the same amount of antibiotic was added as pure powder (as in commercial formulations); moreover the media containing the released antimicrobial drug was capable of preventing the growth of numerous bacteria species responsible for prosthetic joint infections (both catalogue strains and clinical isolates) for longer periods of time than in case of commercial formulation; thus confirming the extended antimicrobial properties of the drug once released from the carrier.

No detrimental effects toward human osteoblasts were also observed; moreover bone cement material characteristics such as curing time, water uptake and mechanical properties were unaffected when the silica nanocarriers were added. 


\section{Introduction}

About 67 thousand and 80 thousand joint replacement surgeries were performed in England and Wales in $2017{ }^{1}$ for hip and knee respectively. These procedures are carried out on a growing number of patients because of the success in improving life quality and because of the aging population, along with obesity one of the risk factors leading to joint damage. Poly-methyl-methacrylate (PMMA) bone cement is commonly employed to fix arthroplasty devices to bones; the use of such material is due to the quick setting time and good mechanical properties of the PMMA bone cement ${ }^{2}$. Post operatory infections can occur after any surgery and they pose additional costs directly to healthcare providers by means of increased medicinal treatments and prolonged hospital stays. They are also a serious risk for the patients as such infections can have life threatening/changing impacts. Infections incidence of about 1-2\% was observed depending on the joint affected by the surgery ${ }^{3,4}$; additionally, infections are more likely to develop after revision than after primary surgery ${ }^{5}$. Revision surgery cost in case of PJI $(\sim 21,000 £)$ is almost twice the cost of the cost of the same procedure in non-infected cases $(\sim 11,000 £)$ because operating times are longer, greater blood losses and higher rates of complications and extended hospitalisation (length of stay) ${ }^{6}$. The overall economic impact of PJI to American health care system had been estimated at \$566 million in 2009 only for the hospital direct costs (therefore neglecting all indirect costs i.e. lost productivity of patients and/or carer and other direct cost such as further GP visits and rehabilitation) with the forecast to reach $\$ 1.62$ billion in $2020^{7}$.

Antibiotics are commonly added to bone cements in order to prevent infections. Bone cement containing gentamicin or tobramycin 2-4\% w/w (Palacos R+G and Simplex with gentamicin) are commercially available and routinely employed in joint replacement surgeries. Antibiotic mixing results in not uniform distribution in the bone cement, leading to an uncontrolled release . Mainly, the antibiotic release occurs during the first few days (about a week) after implantation hence this approach does not protect against the infections that occur in the months after the surgery. ${ }^{5}$ The general consensus is that early infections are caused by bacteria that enter the patient body through the surgical wound (exogenous) and immediately grow resulting in tissue damage; on the contrary late 
infections are assumed to be caused by bacteria that enter the organisms through other access points (haematogenous) i.e. any other minor wounds or orally, considerable time after the surgery and reach the joint replacement device through the blood stream ${ }^{8,9}$. The abiotic surface of the device constitutes a favourable environment to microorganism biofilm formation and growth. Therefore late infection are not caused by microorganisms penetrating the wound during or shortly after surgery and surviving the initial antimicrobial activity of the antibiotic bone cement but caused by bacteria that reach the joint replacement device at a later stage ${ }^{10}$. Hence, the ideal bone cement should provide antimicrobial activity for extended period of time in order to exhibit a prophylactic action against late infection offset; this could be achieved through the application of drug delivery systems ${ }^{11}$. For this purpose antibiotics delivery systems have been prepared through various techniques, for example tethering the drug to a surface ${ }^{12-14}$ or to a particle ${ }^{15-18}$; encapsulating the active molecule in nanoparticles ${ }^{19}$ or liposomes ${ }^{20}$ or depositing the drug using Layer-by-Layer (LbL) assembly ${ }^{21,22}$. Such multitude of approaches demonstrates the clear clinical need of an antimicrobial delivery system for bone cement; however it also reveals the still unsatisfactory performance of the available approaches. For example the entrapment of antibiotic in mesoporous silica coated with hydrolysable polymers suffers the trade-off between extended efficacy and immediate release; in order to extend the release, thicker coatings are required but this, in turn, prevents release in the first hours/days of contact with fluids ${ }^{23}$. Other approaches, like nano-tubes, hydroxyapatite nano-rods or liposomes, despite providing an improved release profile, had detrimental effects on bone cement mechanical properties seriously impinging on the practical application of such technologies ${ }^{24,25}$. Layer-by-Layer self-assembly is a coating technique employing oppositely charges polyelectrolyte adsorbed onto a charged surface alternatively ${ }^{26,27}$. It also widely used in medical devices as it is possible to embed drugs into LbL constructs replacing a polyelectrolyte with the chosen drug or conjugating the drug to one of the polyelectrolytes ${ }^{28,29}$.

We hypothesised that silica nanoparticles containing antibiotics through LbL could extend antibiotic release when embedded in PMMA bone cement. In this work, Layer-by-Layer coatings have been prepared on silica nanoparticles using gentamicin and two different poly-beta-amino-esters (PBAEs). 
Silica nanoparticles are widely used as drug carriers because their blood compatibilitye $e^{30-33}$ and we have shown that it is possible to achieve prolonged (few weeks) gentamicin release from silica nanoparticles coated with gentamicin using LbL while release is sustained for only a few days when conjugation is employed ${ }^{34}$. Gentamicin is aminoglycoside antibiotic with large-spectrum activity ${ }^{35}$ and despite rising resistance, it is still widely used in orthopaedic applications ${ }^{36}$ and embedded into bone cement ${ }^{37}$.

PBAE constitutes a class of positively charged and hydrolysable polyelectrolytes, have been shown to be better suited to prepare LbL constructs capable of sustained gentamicin release for prolonged periods of time compared to other polyelectrolytes such as chitosan ${ }^{34,38}$.

The properties of the nanoparticles have been determined and the release profiles from PMMA bone cement was established. Furthermore, the antimicrobial activity of the released drug has been tested along with the material properties of the nanocomposite bone cement in order to ascertain that the addition of the silica nanocarriers to the PMMA bone cement did not compromise other functional properties of the orthopaedic material.

\section{Materials and Methods}

\subsection{Chemicals}

Gentamicin sulphate, tetraethyl-orthosilicate (TEOS), 3-aminopropyltriethoxysilane (APTS), 1,4 butanediol diacrylate, Piperazine, 4,4'-Trimethylenedipiperidine, ortho-phthaldialdehyde reagent solution (OPA) and sodium alginate (Mw 80.000-120.000 Da)were purchased from Sigma-Aldrich. Triton X-100, ammonium hydroxide (29.6\%), cyclohexane, $n$-hexanol, Dichloromethane (DCM), diethyl-ether, isopropyl alcohol and methanol were purchased from Fisher Scientific.

All chemicals were used as-received.

\subsection{Synthesis silica nanoparticles}

Silica nanoparticles $\left(\mathrm{SiO}_{2}\right)$ were prepared by hydrolysis of TEOS in reverse microemulsion and amino functionalised with APTS..$^{38}$ 


\subsection{Polymer synthesis}

Piperazine or 4,4'-Trimethylenedipiperidine (Sigma, UK) were used as amine monomers. Amino terminated poly( $\beta$-amino ester)s were synthesized by mixing 1,4 butanediol diacrylate and amine monomers in a 1:1.1 ratio in Dichloro-methane (DCM) at a concentration of 5ml of DCM per 3.7 mmol of acrylate. 38

PBAEs prepared using piperazine and 4,4'-trimethylendipiperidine will be referred as POLY1 and POLY2 through the text, respectively (Figure 1a).

POLY1

(a)<smiles>C=CC(=O)OCCCCOC(=O)C=[18O]</smiles><smiles>C=CC(=O)OCCCCOC(=O)C=[C+]=CO</smiles>

(b)

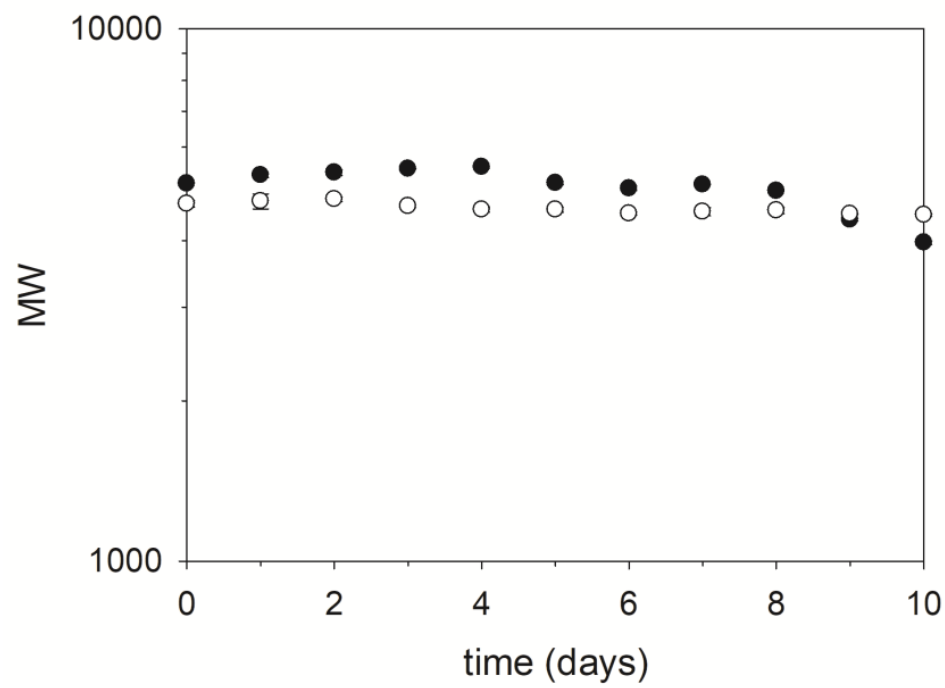

Figure 1. (a) Reaction scheme and structure of POLY1 and POLY2. (b) PBAE hydrolysis in PBS for POLY1 $(\bigcirc)$ and POLY2 (O). 


\subsection{Gel permeation chromatography (GPC)}

The determination of the kinetics of hydrolysis of POLY1 and POLY2 was carried out through the reduction of molecular weight as determined by the FPLC (Fast Protein Liquid Chromatography) using Akta Design (Amersham pharmacia biotech-Sweden) supplied with Superdex 75 10/3000 GL column and acetate buffer $\mathrm{pH} 5(1 \mathrm{ml} / \mathrm{min})$ as mobile phase. ${ }^{38}$

PBAEs solution $(10 \mathrm{mg} / \mathrm{ml})$ in PBS (pH 7.4) were prepared and incubated statically at $37^{\circ} \mathrm{C}$; samples $(0.5 \mathrm{ml})$ were collected and immediately analysed over 30 days. For each PBAE, two replicates were taken. A calibration curve was prepared using 7 standards with known molecular weights (purchased from Fluka Chemie AG and Polymer Laboratories Ltd) to correlate MW and retention volumes. ${ }^{38}$

\subsection{Layer-by-Layer deposition}

Gentamicin containing coatings were prepared on silica nanoparticles using the process described by Al Thaher et al. ${ }^{38}$ for each of the compounds employed The particles were coated with 5 quadruple layers, deposited onto a glass watch and allowed to dry in a fume hood.

\subsection{Silica-antibiotic nanocarriers characterisation}

\subsubsection{Transmission electron microscopy (TEM)}

Size and shape of the nanoparticles were determined through transmission electron microscopy (TEM); $4 \mu 1$ droplet of conjugates suspension were deposited on a plain carbon-coated copper TEM grid, water was evaporated under ambient laboratory conditions for several hours. Bright field TEM images were obtained using a TEM (Philips CM12, FEI Ltd, UK) operating at 80kV fitted with an Xray microanalysis detector (EM-400 Detecting Unit, EDAX UK) utilising EDAX's Genesis software. Images (magnification of the images was x 100000) were recorded using a SIS MegaView III digital camera (SIS Analytical, Germany) and analysed with ImageJ; the diameter of at least 100 particles was determined for each type of nanoparticles. ${ }^{38}$ 


\subsubsection{Thermogravimetric analysis (TGA)}

Thermogravimetric analysis (TGA) was performed using a Stanton Redcroft, STA-780; data were recorded from 25 to $600{ }^{\circ} \mathrm{C}$ with a constant heating rate of $10{ }^{\circ} \mathrm{C} \min ^{-1} \cdot{ }^{38}$

\subsubsection{Zeta potential}

Approximately $2 \mathrm{mg}$ sample were dispersed in $1 \mathrm{ml}$ of acetic buffers $(0.1 \mathrm{M})$ at $\mathrm{pH}$. The suspension was vortexed and transferred to a capillary cell and the zeta potential was measured immediately using Zetasizer ZEN 3600, Nano Series (Malvern, UK). ${ }^{38}$

\subsubsection{Gentamicin release}

$10 \mathrm{mg}$ of NPs were incubated in $1 \mathrm{ml}$ sterile PBS at $37^{\circ} \mathrm{C}$. Every day all media was replaced with fresh sterile PBS. Gentamicin contained in the release media was quantified thorough fluorescence spectroscopy using $o$-phthaldialdehyde (OPA); in a black 96 wells plate, $70 \mu \mathrm{l}$ of media containing antibiotic were mixed with $70 \mu \mathrm{l}$ of iso-propanol and $70 \mu \mathrm{l}$ of OPA reagent; after $30 \mathrm{~min}$ at room temperature in the dark, the fluorescence was determined (excitation wavelength $=340 \mathrm{~nm}$ and emission wavelength $=450 \mathrm{~nm}$ ) with a fluoroscan (FLUOROstar Optina, BMG Labtech); standards of known gentamicin concentration were also analysed simultaneously to provide calibration. ${ }^{38}$ All characterisations were carried out on nanoparticles obtained from at least three independent batches; results are presented as mean \pm standard deviation.

\subsection{Bone cement preparation}

Bone cement preparation was carried out according to manufacturer and ISO5833:2002 (Implants for surgery-Acrylic resin cements) instructions. All the contents of the bone cement were stored at recommended conditions and conditioned to room temperature for 2 hours before mixing. The solid component (poly-methyl-methacrylate $(83.8 \% \mathrm{w} / \mathrm{w}) ; \mathrm{BaSO}_{4}(10.0 \% \mathrm{w} / \mathrm{w})$; benzoyl peroxide $(1.9 \% \mathrm{w} / \mathrm{w})$ and the liquid component (methyl-methacrylate $(97.5 \% \mathrm{w} / \mathrm{w})$ and N,Ndimethyl-p-toluidine $(2.5 \% \mathrm{w} / \mathrm{w})$ were weighed separately. Gentamicin sulphate $(4 \% \mathrm{w} / \mathrm{w})$ or coated silica nanoparticles $(15 \%$ w/w) were added to the solid phase. Finally, both components were hand 
mixed (solid to liquid ratio 2.1:1) in a polypropylene bowl with a polypropylene spatula for 1 minute, before being filled into a cylindrical mold ( $6 \mathrm{~mm}$ diameter and $12 \mathrm{~mm}$ height). After applying the cement, the mold was pressed between two steel endplates covered with PTFE film. After 2 hours, the samples were pushed out of the mould using a steel rod and rough edges were sanded down with silicon carbide paper ( 320 grit). Samples were allowed to cure for $24 \pm 2$ hours at $23^{\circ} \mathrm{C}$ prior further experiments.

\subsection{Bone cement characterisation}

\subsubsection{Gentamicin release}

Bone cement cylinders were incubated in $3 \mathrm{ml}$ sterile PBS at $37^{\circ} \mathrm{C}$. Every day all media was replaced with fresh sterile PBS. Gentamicin contained in the release media was quantified thorough fluorescence spectroscopy using $o$-phthaldialdehyde (OPA); in a black 96 wells plate, $70 \mu$ of media containing antibiotic were mixed with $70 \mu \mathrm{l}$ of iso-propanol and $70 \mu \mathrm{l}$ of OPA reagent; after $30 \mathrm{~min}$ at room temperature in the dark, the fluorescence was determined (excitation wavelength $=340 \mathrm{~nm}$ and emission wavelength $=450 \mathrm{~nm}$ ) with a fluoroscan (FLUOROstar Optina, BMG Labtech); standards of known gentamicin concentration were also analysed simultaneously to provide calibration. ${ }^{38}$ All characterisations were carried out on nanoparticles obtained from at least three independent batches; results are presented as mean \pm standard deviation.

\subsubsection{Antimicrobial activity}

Clinical isolates (species determined through PCR) and catalogue strains (S. aureus NCIMB 9518, $S$. aureus ATCC 25923, S. aureus ATCC 9144, S. epidermidis ATCC 12228, MRSA NCTC 12493, S. pyogenes ATCC 12344, E. coli NCTC 14418, P. aeruginosa PA01, A. baumannii NCIMB 9214) of bacteria commonly associated with prosthetic joint infections were stored on BHI Agar plates at $4{ }^{\circ} \mathrm{C}$. A loopful of cells was used to inoculate $10 \mathrm{ml}$ of BHI broth that was then incubated at $37^{\circ} \mathrm{C}$ statically for 24h. The bacteria suspension was diluted in fresh sterile BHI broth (1:1000) and used to inoculate the bone cement release medium after each day of antibiotic release; in a 96 wells plate $20 \mu \mathrm{l}$ of 
diluted cells suspension were diluted with $100 \mu \mathrm{l}$ of release buffer. Assessment of bacteria growth was performed after incubation for 24 hours at 37 degree on three independent cell cultures and triplicate release buffers. Results are presented as the day associated to the last release medium capable of preventing bacterial growth.

\subsection{Cytocompatibility}

Human osteoblast cells (Saos-2) ATCC HTB-85 were cultured in RPMI-1640 medium as previously described. ${ }^{39}$

Bone cement samples were sterilised with $70 \%$ alcohol and washed three times with sterile PBS prior to use. Samples were placed in 24 wells plates containing $2 \mathrm{ml}$ of osteoblast cells suspension prepared as described above. Osteoblasts were incubated with the bone cement at $37{ }^{\circ} \mathrm{C}$ in humidified atmosphere with $5 \% \mathrm{CO}_{2}$ for up to 21 days changing medium twice a week.

\subsubsection{MTT}

Osteoblast were incubated on the bone cement samples for 2 days then they were transferred to a clean 24 well plate with $2 \mathrm{ml}$ of fresh medium without red phenol. Osteoblast cells viability was assessed using the MTT assay (Sigma, UK) as described in Perni et al. ${ }^{39}$ Results are presented as the average and standard deviation of 3 independent bone cement samples.

\subsubsection{Osteoblast mineralisation activity (Alizarin Red A)}

Osteoblast were incubated on the bone cement samples for 21 days, when the medium was removed from all wells. After fixing the cells with glutaraldehyde, alizarin red staining (ARS) was carried according to the procedure described in Tommasi et al. ${ }^{39}$ All mineralisation tests were performed in triplicates.

\subsubsection{Microscope imaging}

Saos- 2 cells were cultured on the bone cement samples for 4 days; after this time cells were washed thoroughly three times in PBS. Cells were then fixed with $3.7 \%(\mathrm{w} / \mathrm{v})$ formaldehyde in PBS at room temperature for $5 \mathrm{~min}$ and permeabilised with $0.1 \%$ Triton $\mathrm{X}-100$ at room temperature for $5 \mathrm{~min}$. Then cells were stained with $50 \mathrm{mg} / \mathrm{L}$ of tetramethyl rhodamine B isothiocyanate-conjugated phalloidin 
(Sigma-Aldrich, St. Louis, MO, USA) for 40 minutes at room temperature, followed by incubation with $5 \mathrm{mg} / \mathrm{l}$ of trihydrochloride Hoechst 33342 (Thermo Fisher Scientific, Eugene, OR, USA) for 10 minutes in the dark. After washing with PBS, samples were mounted and examined using LSM 880 upright confocal laser scanning microscope with Airyscan (Zeiss, Oberkochen, Germany) for visualization of the staining with a $63 \mathrm{X}$ magnification objective. Processing of the obtained images was conducted using ZEN imaging software (Zeiss).

\subsection{Material properties of bone cement containing nanocarriers}

\subsubsection{Bone cement curing time}

Rheological measurements were performed using a strain controlled rheometer, ARES (TA Instruments) equipped with $8 \mathrm{~mm}$ diameter plates.

Analysis was carried out in the linear regime by performing dynamic time sweep tests at constant frequency $(1 \mathrm{rad} / \mathrm{sec})$ and strain $(0.1 \%)$. Tests were conducted at controlled room temperature. For all tests, the bone cement solid phase, with gentamicin $(2 \% \mathrm{w} / \mathrm{w}$ or equivalent amount contained in developed Si nanocarriers), was mixed with the liquid phase, quickly with a spatula; the mixture was deposited onto the lower plate and experiments were started as fast as possible. To account for the time elapsed during mixing and pouring, a timer was started at the moment of mixing the liquid with powders. Measurement of complex Young modulus and phase angle were taken every $6 \mathrm{~s}$ for up to 15 $\min$.

\subsubsection{Water uptake}

Bone cement cylinders were weighted before being employed in gentamicin release studies and after each day when the release media was replaced.

\subsubsection{Compressive and bending strength}

Compression and bending tests were undertaken on the Zwick Roell ProLine table-top Z050/Z100 materials testing machine according to BS ISO 5833:2002. The compression tests were conducted at a constant cross-head speed of $20.0 \mathrm{~mm} / \mathrm{min}$ to produce a curve of displacement against load. For each specimen, the compressive strength of the bone cement was determined by dividing the force 
applied to cause fracture by the original cross-sectional area of the cylinder. Bending modulus and bending strength were estimated according to BS ISO 5833:2002.

The results are presented in all cases as average of five specimens \pm standard deviation.

\subsubsection{Fracture toughness}

Fracture toughness of cement samples was determined according to ISO13586:2000. The bone cement samples were rectangular $($ Length $=45 \pm 0.1 \mathrm{~mm}$, Width $=10 \pm 0.1 \mathrm{~mm}$ and Depth $=3.3 \pm 0.1 \mathrm{~mm}$. A sharp razor blade was used to create a V-shaped notch of 4.4-5.5 $\mathrm{mm}$ at the centre of the sample. The rectangular sample was loaded incrementally using a Zwick Roell ProLine table-top Z050/Z100 materials testing machine (Zwick Testing Machines Ltd., Herefordshire, UK). The fracture toughness is a three-point test, where the distance between the rollers is $40 \mathrm{~mm}$. The length of the crack was measured by a Pye Scientific travelling microscope (Pye Scientific, Cambridge, UK), and the width and length of each sample was measured by a Vernier calliper.

The results of critical stress intensity and critical energy are presented as mean of five samples.

\subsection{Statistical analysis}

The comparison of the effects of Si nanoparticles on Saos-2 cells metabolic activity (viability and alizarin Red A assay) were performed using ANOVA test.

The comparison of nanocarriers on the release profiles was carried out fitting a generalised linear mixed model after logarithmic transformation of the gentamicin concentration values using the identity link function; followed by ANOVA test and Tukey's post hoc test of individual pair of data sets $(p<0.05)$. Multivariate analysis of variance (MANOVA) was carried out to test the antimicrobial activity of nanocarriers against the commercial formulation, where the multiple dependent variables were the number of days the growth was prevented, using the Pillai test. Paired-wise ANOVA comparison was further performed to determine the impact on individual species.

All statistical analysis was performed using R (R Core Team, 2012) and lme4 package ${ }^{40}$. 


\section{Results}

\subsection{Polymer kinetics of hydrolysis}

No appreciable reduction of polymer MW accountable to hydrolysis in PBS of both PBAE was detected for up to 10 days (Figure 1b).

\subsection{Silica nanoparticles properties}

\subsubsection{Size}

The silica nanocarriers were spherical and with a diameter of about $50 \mathrm{~nm}$ and a Gaussian distribution (Figure $2 \mathrm{a}$ and $\mathrm{d}$ ), the deposition of 5 quadruple layers through LbL did not impact the particles diameter regardless of the PBAE used (Figure $2 \mathrm{a}, \mathrm{b}$ and $\mathrm{c}$ ).

\subsubsection{Zeta potential}

The amino functionalised nanoparticles had a positive charge (Figure $2 \mathrm{e}$ ) of $+22 \mathrm{mV}$; during LbL the zeta potential of the particles after each layer deposited had a see-saw profile. After the deposition of alginate, the particles had negative charge of about $-30 \mathrm{mV}$ while after the deposition of gentamicin the charge was slightly positive $(+4 \mathrm{mV})$. When any of the PBAEs tested (POLY1 or POLY2) were layered on the nanoparticles surface the zeta potential became $+8 \mathrm{mV}$. 

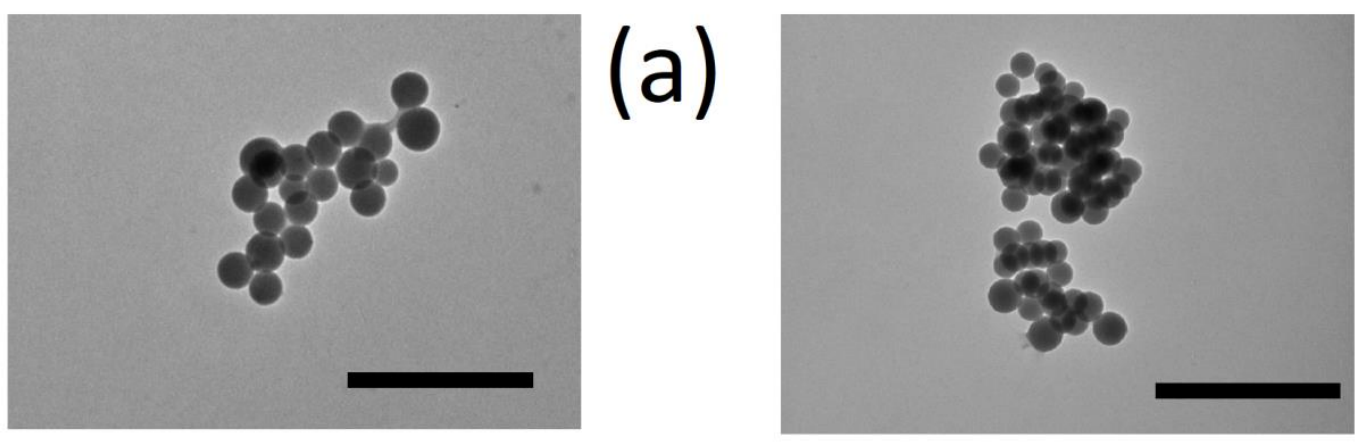

(b)

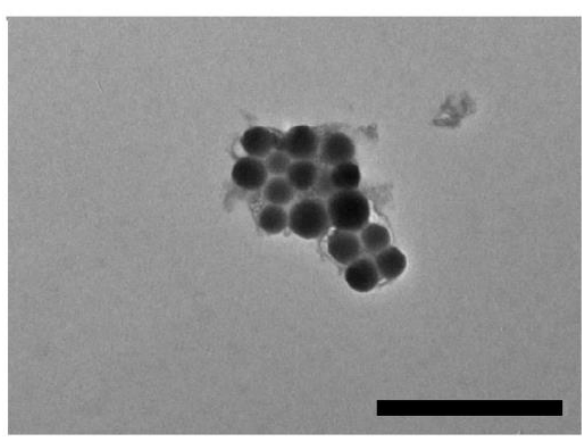

(c)

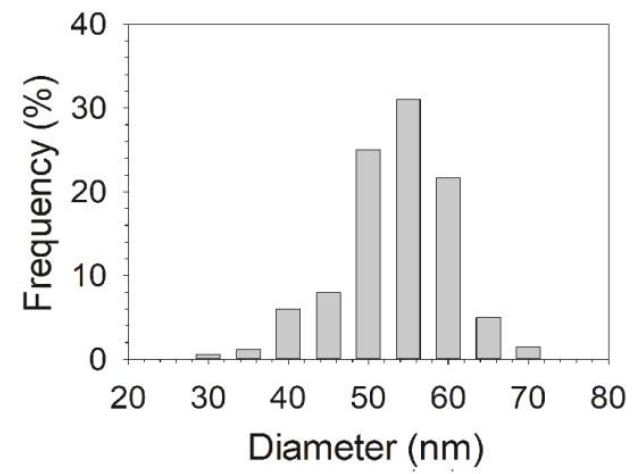

(d)

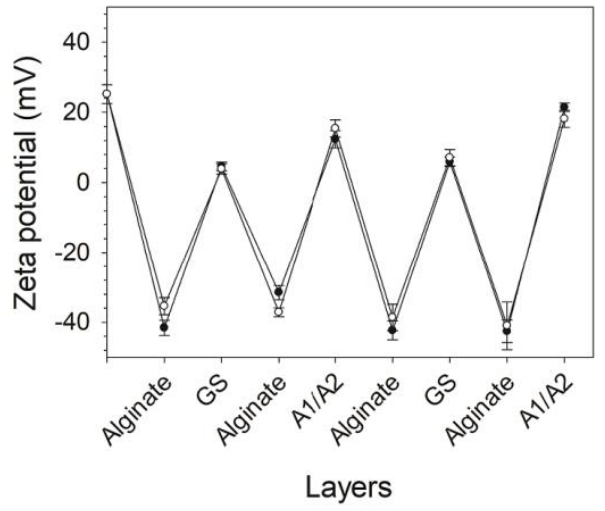

(e)

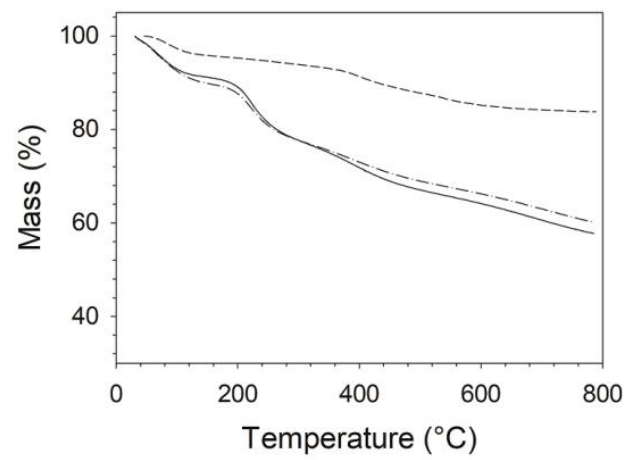

(f)

Figure 2. Examples of TEM images (bar equivalent to $500 \mathrm{~nm}$ ) for uncoated Silica nanoparticles (a) or after deposition of 5 quadruple layers using POLY1 (b) and POLY2 (c); size distribution of uncoated silica nanocarriers (d). Zeta potential after each deposited layer on silica nanoparticles for the first two quadruple layers (e) $\bigcirc$ for POLY1 and $\bigcirc$ for POLY2 (f) Examples of thermograms for amino functionalized silica nanoparticles (dashed line) and silica nanoparticles layered with 5 quadruple layers of alginate - gentamicin - POLY1 (solid line) - alginate or alginate - gentamicin POLY2 (dash-dot line) - alginate. 


\subsubsection{TGA}

The thermograms of the silica nanoparticles revealed a mass loss with increasing temperature reaching a plateau at about $700^{\circ} \mathrm{C}$ (Figure $2 \mathrm{f}$ ); furthermore the amount of deposited layers can be determined as the difference between the mass loss of the coated and uncoated nanoparticles. TGA showed that the organic content (due to LbL deposition) was comparable for the two PBAEs used and represented approximately $25 \% \mathrm{w} / \mathrm{w}$ of the prepared nanocarriers.

\subsection{Gentamicin release}

Gentamicin release kinetics from the coated nanoparticles was highest at the beginning and monotonically decreased down to 0 after about 25 days for both polyelectrolytes used; nanoparticles coated through LbL using POLY1 had an overall higher released and corresponding loading (Figure $3 a)$; the estimated antibiotic loading on the nanocarriers was $\sim 12$ and 10\% w/w for POLY1 and POLY2 respectively. Gentamicin release from the bone cement was highest at the beginning; the greatest amount of gentamicin was released from the PMMA bone cement containing the drug in its pure form (Figure 3b) and representing a commercial formulation of antibiotic laden bone cement. However, the release of the antibiotic was almost finished after about 5-6 days when gentamicin was simply mixed in the PMMA dough. On the other hand, for the antibiotic was deposited on silica nanoparticles using the LbL technique and PBAEs, gentamicin was released from the bone cement samples for about 25 days (Figure 3b). When POLY2 was employed as polyelectrolyte, gentamicin release was greater during the first 2-3 days than POLY1 but the overall amount of drug released at the end of the release period (30 days) was greater for POLY1.

Fitting of the release profile with a mixed effect model (Table S1) revealed that when POLY1 or POLY2 were employed gentamicin release kinetics followed a first order model (Figure S1) moreover visual inspection of residual plots did not reveal any obvious deviations from homoscedasticity or normality (Figure S2). On the contrary the commercial formulation release profile was not well fitted by this release model as clearly evident in the plots of residuals (Figure S2). Moreover, the release kinetic from Si nanocarriers prepared using POLY1 was lower than for POLY2 (p <0.00034) and both 
were lower than the commercial formulation ( $\mathrm{p}<0.0001)$.
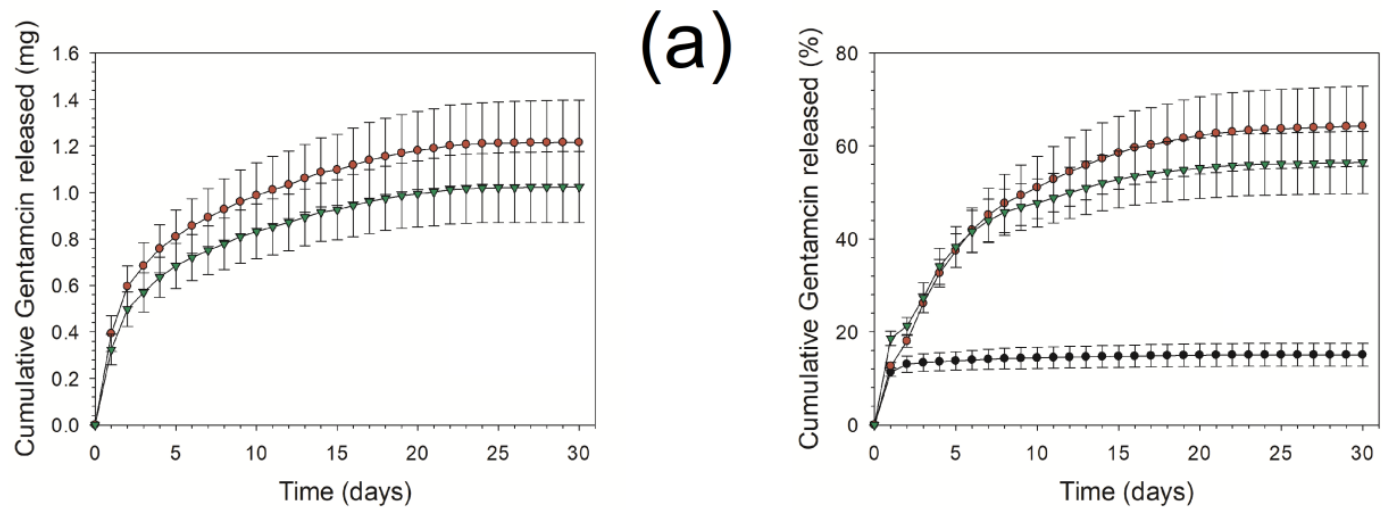

(b)

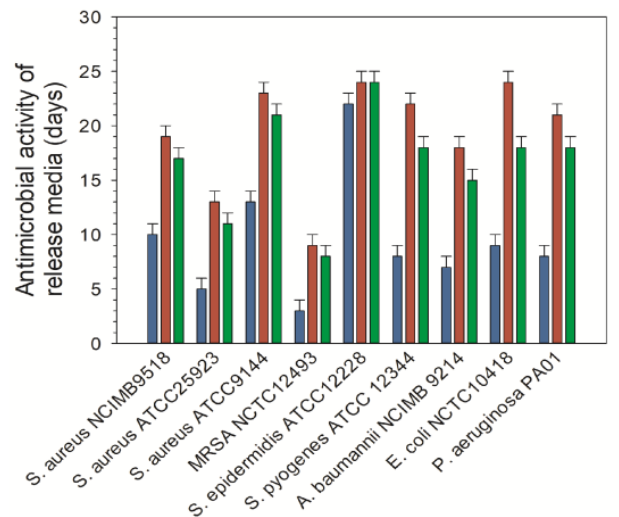

(c)

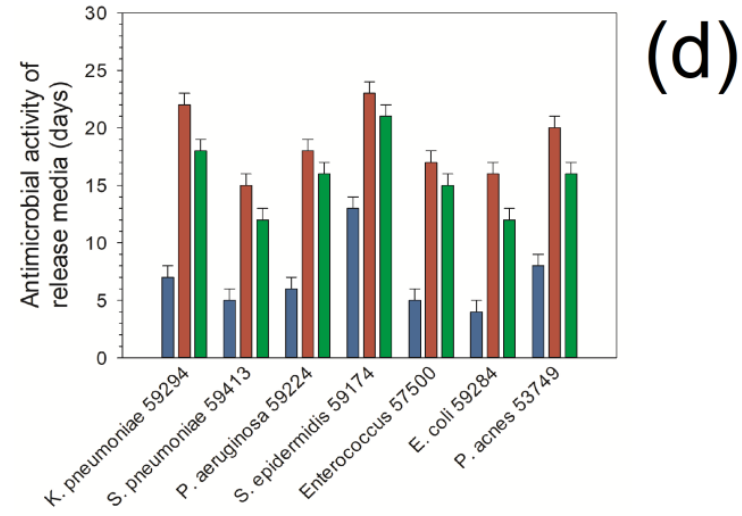

Figure 3. Cumulative release of gentamicin from coated silica nanoparticles (a), PMMA bone cement as $\%$ of the initial amount of antibiotic (b) when added as pure powder $(0)$ or deposited on silica nanocarriers through LbL using POLY1 $(\bullet)$ or POLY2 $(\nabla)$. Number of days of antimicrobial activity of the release media of PMMA bone cement containing gentamicin when added as pure

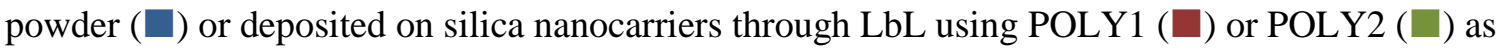
polyelectrolyte for catalogue bacterial strains (c) and clinical isolates of prosthetic joint infections (d).

\subsection{Antimicrobial activity}

The antimicrobial activity of the bone cement was tested by inoculating the buffer containing the released drug with various bacteria species. The release buffer was changed daily, therefore, each day the amount of drug contained was representative of the release kinetic after that certain period of time. The antibiotic released from the PMMA bone cement matrix decreased monotonically with increasing contact time with the buffer; hence, at some point, it inevitably fell below the minimum inhibitory 
concentration for a specific species. The longer the antibiotic was released, the longer the buffer was capable of preventing bacterial growth. For example, if no growth was recorded in the buffer collected after 14 days of release but growth was noticed in the buffer collected the following day (after 15 days), it was said that the bone cement was capable of preventing the growth of the specific species for 14 days.

The bacterial tested had species specific sensitivity to gentamicin (Table S2 and S3), and the bone cement exhibited antimicrobial activity against all the species tested. The more resistant species from the catalogues strains employed was MRSA (only prevented from growing by buffers corresponding to a release time < 3days) while the more sensitive was S. epidermidis that was inhibited even by a release buffer collected after 24 days (Figure 3c).

A similar pattern (gentamicin deposited on Si through LbL providing extended antimicrobial activity compared to pure form) was observed also for clinical isolates of prosthetic joint infections (PJI), even though the isolates were generally more resistant to gentamicin than the catalogue strains (Figure 3d). Moreover, not antimicrobial activity was observed from bone cement samples containing coated nanoparticles using only polyelectrolytes.

For each species the release buffer from PMMA bone cement containing pure gentamicin gave the shortest antimicrobial activity for all bacteria tested ( $\mathrm{p}<0.05)$ apart S. epidermidis ATCC 12228 as revealed by MANOVA and subsequent ANOVA on specific species; on the contrary, the bone cement containing the antibiotic deposited through LbL using POLY1 gave the longest antimicrobial effect (Figure $3 \mathrm{c}$ and d). MANOVA performed on the antimicrobial outcome of POLY1 and POLY2 nanocarriers added to PMMA bone cement revealed that Si nanocarriers prepared using POLY1 provided an antimicrobial activity significantly different $(\mathrm{p}<0.05)$ from Si nanocarriers prepared using POLY2 only for the following species: S. pyogenes ATCC 12344; A. baumannii NCIMB 9214, E. coli NCTC 10418; P. aeruginosa PA01; K. pneumoniae 59294; S. pneumoniae 59413; E. coli 59284; P. acnes 53749. 


\subsection{Bone cement properties}

\subsubsection{Water uptake}

Water uptake by the bone cement sample was determined up to 1 month of contact with fluid. The greatest uptake was recorded during the first day of immersion in the buffer and gradually the amount of fluid contained in the samples stabilised, reaching a plateau after about 7 days $\left(\sim 13 \mathrm{~h}^{0.5}\right)$; no difference was found between the PMMA bone cement samples containing pure gentamicin or the silica nanocarriers (Figure 4).

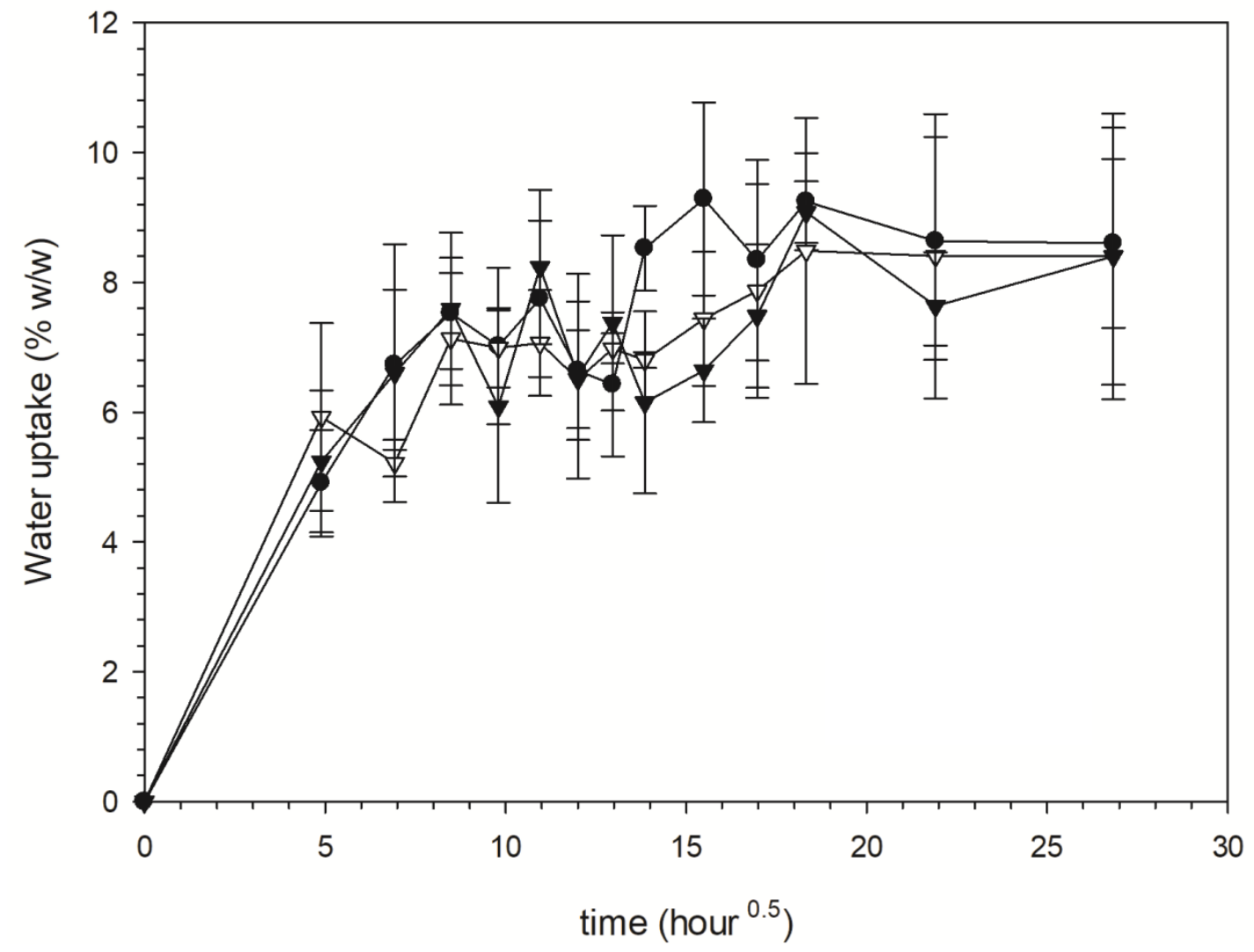

Figure 4. Water uptake of PMMA bone cement with added gentamicin as pure powder $(\bullet)$ or deposited on silica nanocarriers through LbL using POLY1 $(\nabla)$ or POLY2 $(\boldsymbol{\nabla})$ as polyelectrolyte. 


\subsubsection{Bone cement settling time}

Bone cement polymerisation was analysed through rheology, determining the variation of the material properties with time. For all types of bone cement tested (commercial formulation with gentamicin or added Si NP), initial values of the storage modulus (G') and loss modulus (G',) were comparable and the ratio tan delta (G"/G') was about 1 . During sample curing G' showed a monotonical "S-shaped" increase after mixing, reaching a final plateau value of about $10^{8} \mathrm{~Pa}$. The loss modulus (G”) exhibited an initial slow increase, followed by a quick local maximum corresponding to the faster growth in G', finally decreasing to a plateau value (about $10^{6} \mathrm{~Pa}$ ) (Figure 5). The corresponding tan delta (G'/G') profile exhibited a general decline with a local maximum corresponding to the inflection in G'. The time required by the bone cement to cure was chosen as the point of local maximum in tan delta. Commercial formulation required 8.92 min (Figure 5d) and the addition of any of the Si NP based gentamicin delivery system impact on the curing time of the bone cement was not statistically significant $(\mathrm{p}>0.05)$.

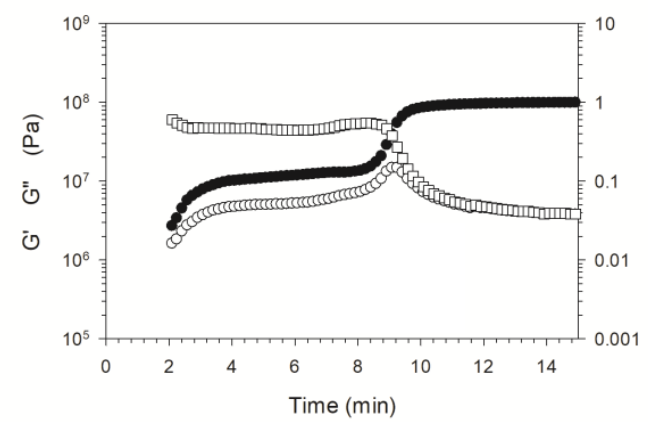

(a)

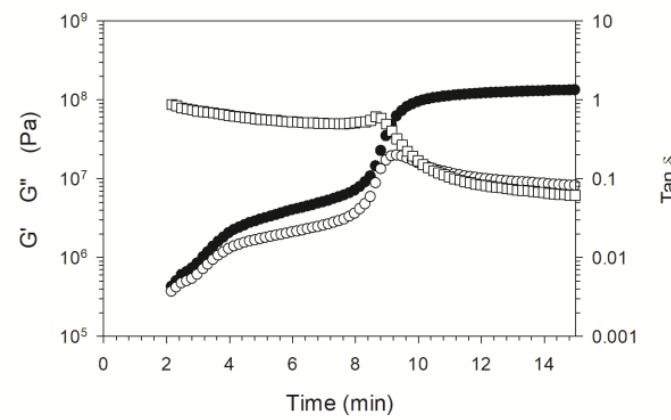

(b)

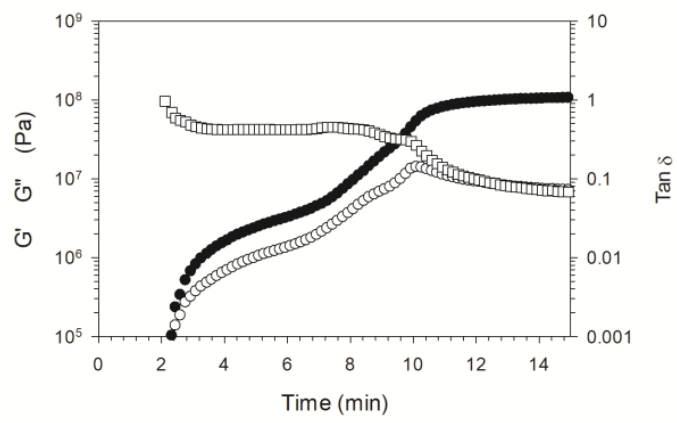

(c)

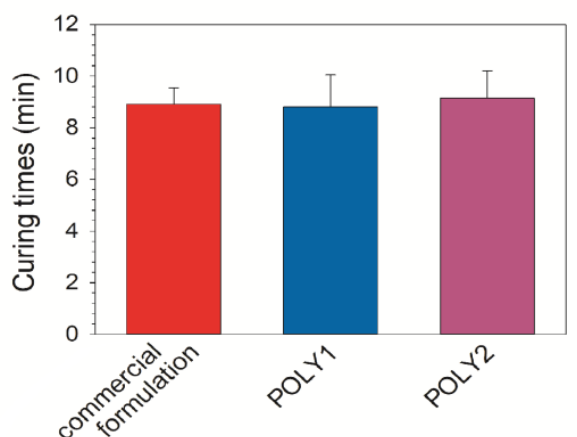

(d)

Figure 5. Time dependent rheological measurements during curing for bone cement with added gentamicin as pure powder (a) or deposited on silica nanocarriers through LbL, by using POLY1 (b) 
or POLY2 (c) as polyelectrolyte, G' $(\bullet), G^{\prime \prime}(\bigcirc)$ and tan delta $(\square)$. (d) Curing times in minutes (mean and standard deviation) of PMMA bone cement with and without added nanoparticles.

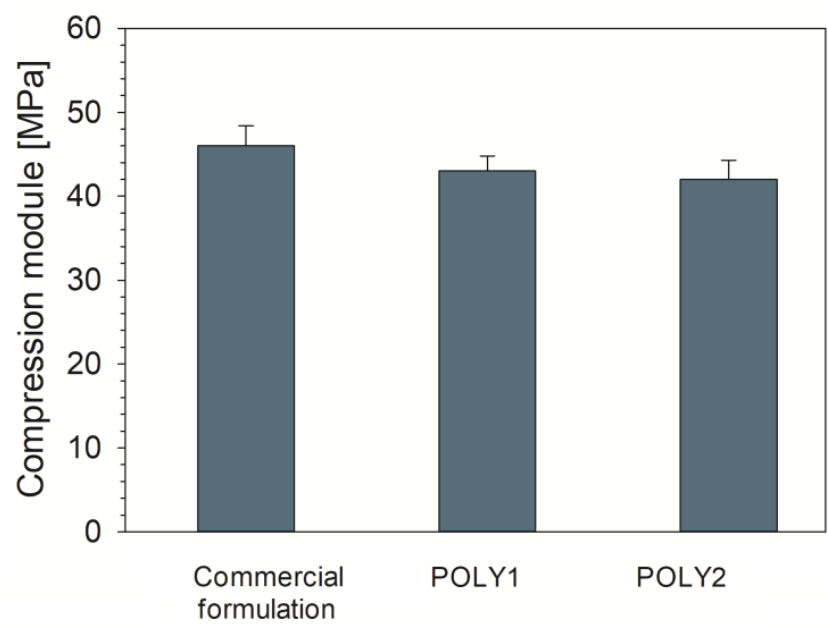

(a)

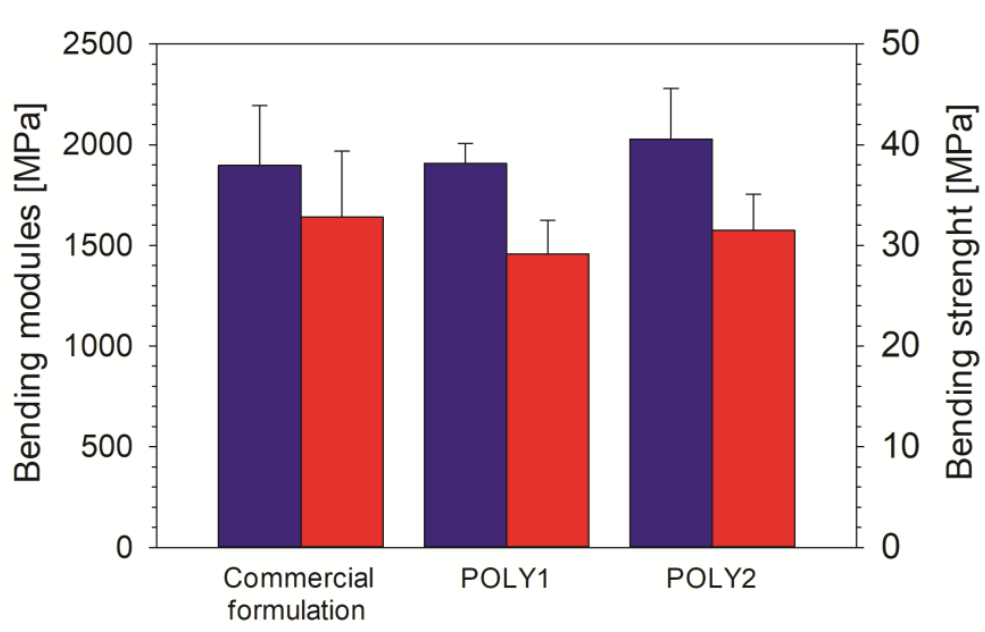

(b)

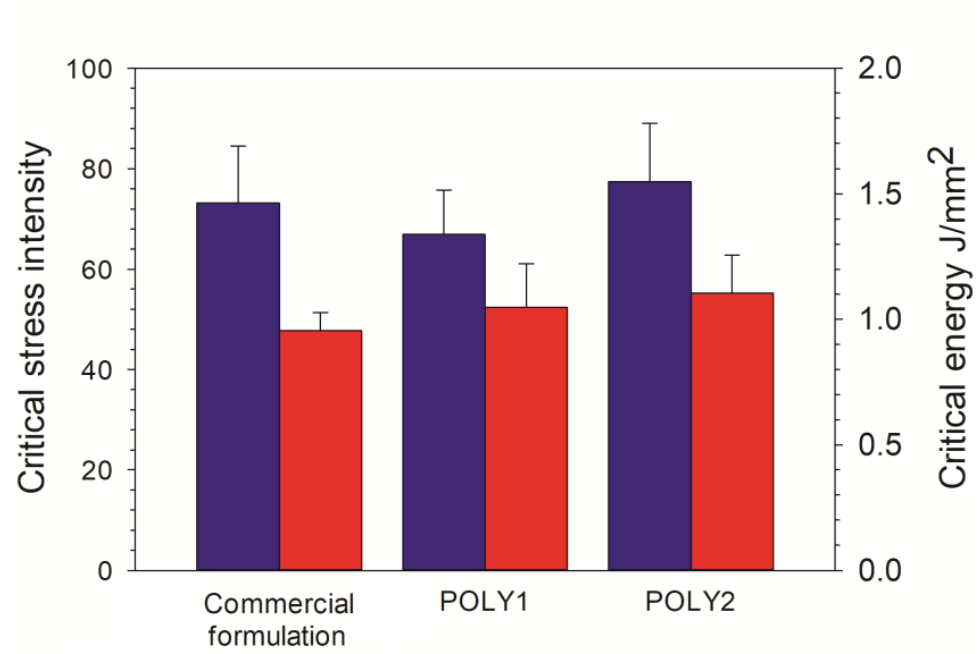

(c) 
Figure 6. (a) Compressive strength ( $\square$ ); (b) bending modulus ( $\square$ ) and bending strength ( $\square$ ) and (c) Critical stress intensity ( $\square$ ) and critical energy ( $\square$ ) of PMMA bone cement with added gentamicin as pure powder or deposited on silica nanocarriers through LbL using POLY1 or POLY2.

\subsubsection{Mechanical properties}

Comparison of compressive and bending properties of the PMMA bone cement with added nanocarriers against bone cement with the same amount of gentamicin in powder form did not reveal any adverse effect of the silica nanoparticles (Figure 6), similarly resistance against fracture propagation was not affected; moreover, these values were matching the requirement for applicability as PMMA bone cement ${ }^{32}$.

\subsubsection{Osteoblast cytocompatibility}

Human osteoblast grown on the commercial formulation or on bone cements containing the silica nanocarriers had the same viability (assessed through MTT) and calcium production (Alizarine Red) as shown in (Figure 7). Moreover, the conformation of the actin filaments of Saos-2 cells grown on bone cement containing the developed nanocarriers did not differ from that of cells seeded on the commercial formulation containing gentamicin powder (Figure 8). 
(a)

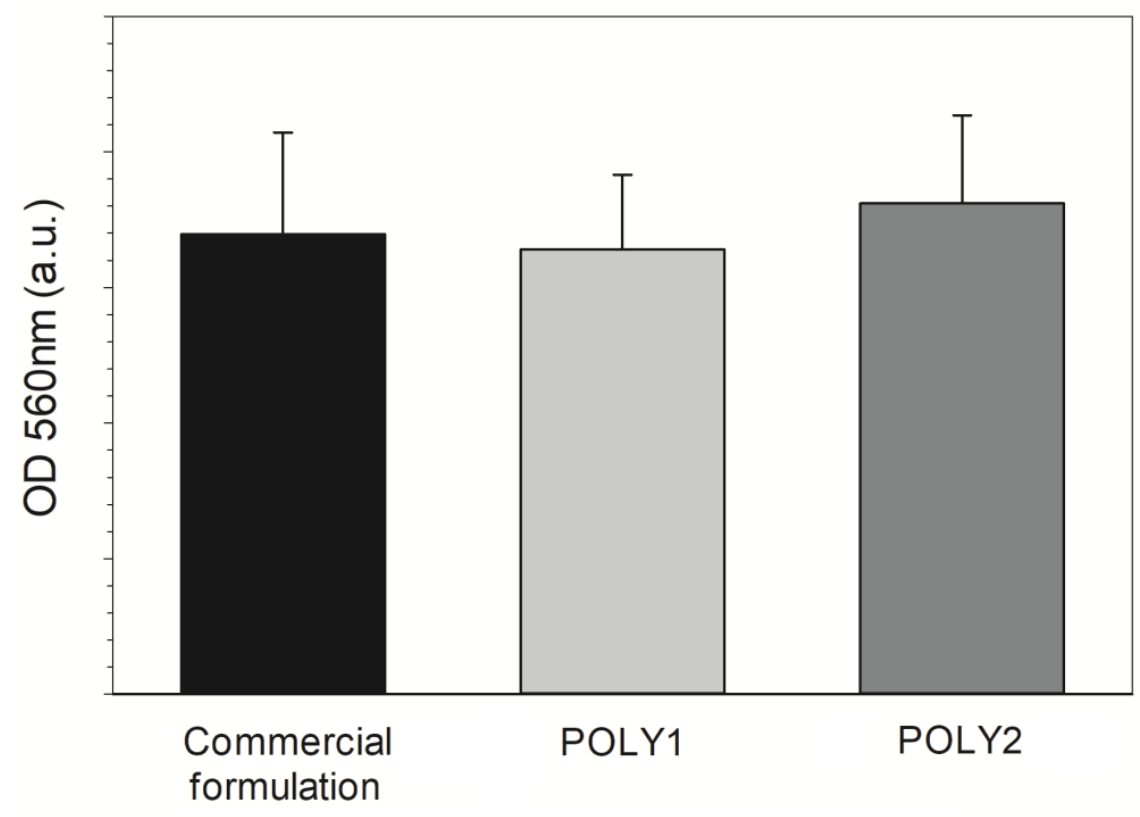

(b)

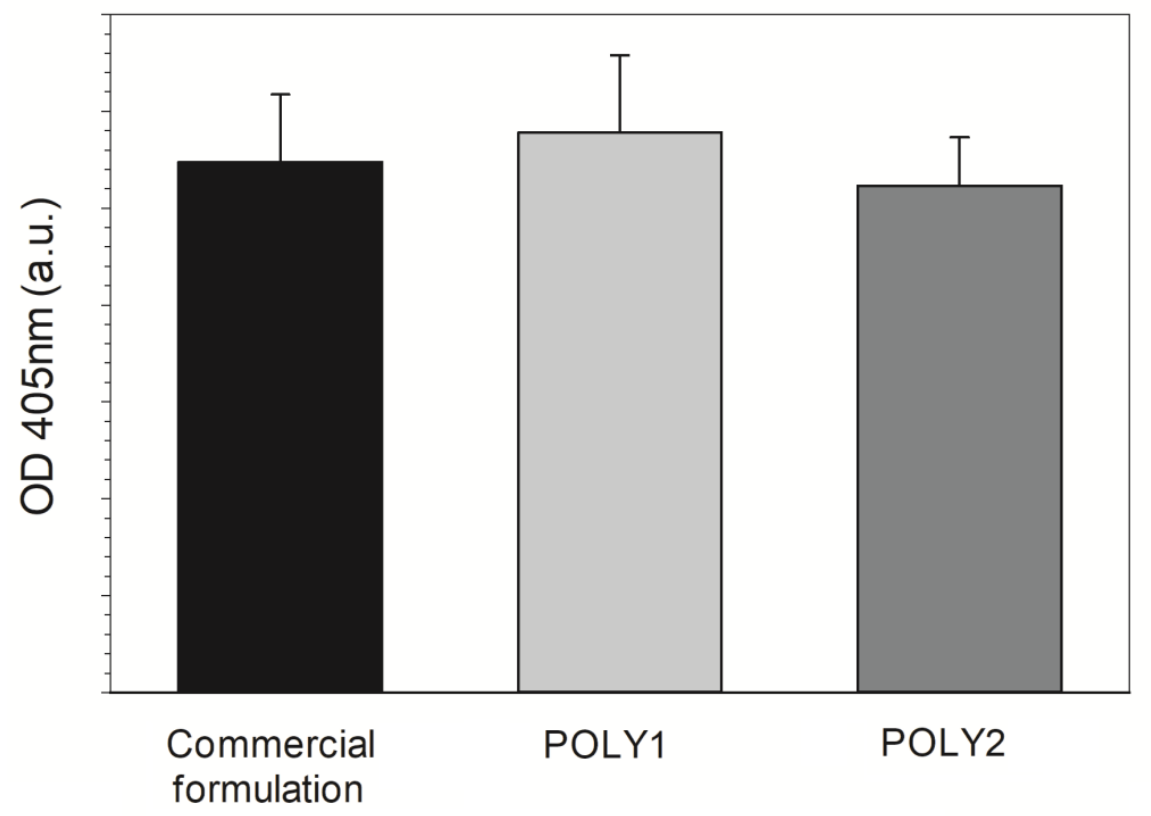

Figure 7. MTT (a) and Alizarin Red A (b) assay of Saos-2 (human osteoblast) cells grown on PMMA bone cement containing gentamicin as pure powder $(\mathbf{\square})$ or deposited on silica nanocarriers through LbL using POLY1 ( $\square$ ) or POLY2 ( $\square$ ) as polyelectrolyte. 
(a)

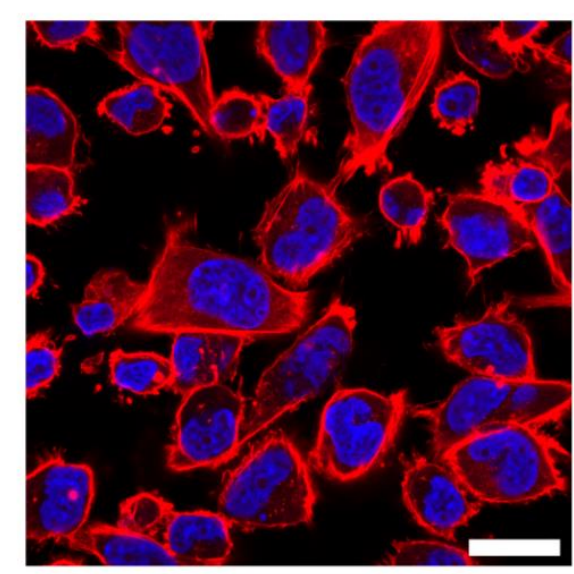

(b)
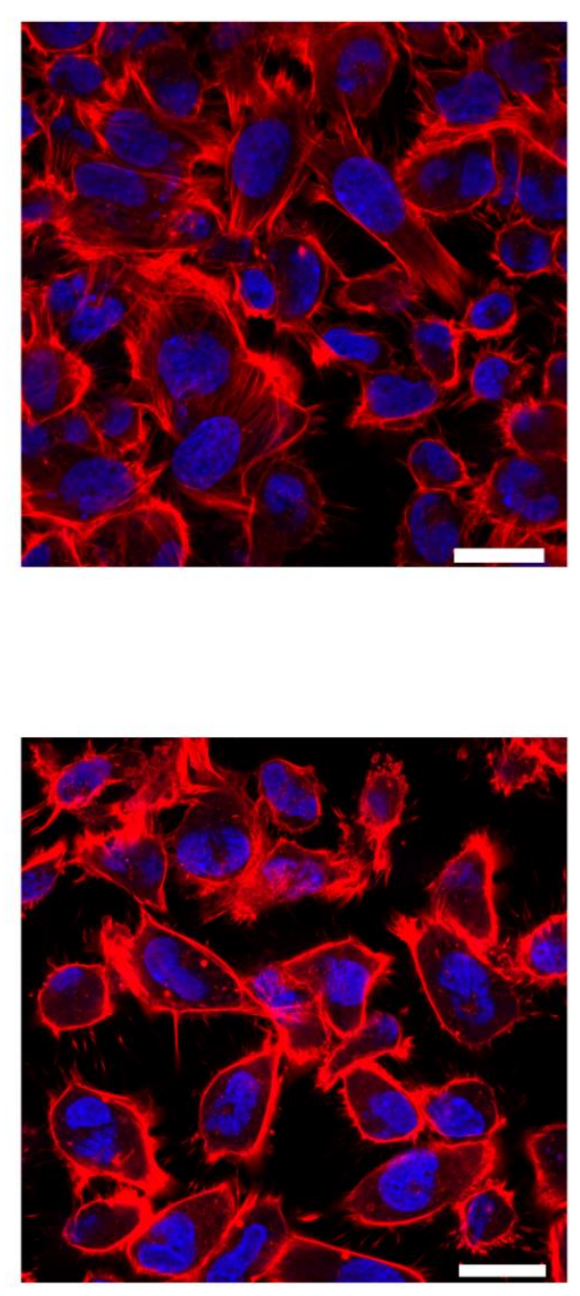

(c)

Figure 8. Microscope images of Saos-2 cells, grown on PMMA bone cement containing gentamicin as pure powder (a) or deposited on silica nanocarriers through LbL using POLY1 (b) or POLY2 (c) as polyelectrolyte, stained with phalladolin (actin) in red and DAPI in blue exposed. bar represent 10 $\mu \mathrm{m}$. 


\section{Discussion}

In our work we took an holistic approach to the development of a drug delivery system for PMMA bone cement using gentamicin as a model drug in virtue of its wide use for this purpose; first we characterised the nanocarriers, then we assessed the release profile to ascertain that the drug release profile was sustained for up to a month when the nanocarriers were embedded in the actual PMMA and that the antimicrobial activity of the antibiotic was retained and finally we verified that no other properties of the PMMA bone cement were negatively impacted by the inclusion of the drug delivery system into the PMMA bone cement matrix.

\subsection{Silica nanoparticles properties}

The silica nanocarriers characterisation was carried out through TGA that is routinely employed to determine the fraction of organic material in a sample as the mass loss during heating is associated to the combustion of the organic molecules present. The mass loss observed in the $\mathrm{Si}-\mathrm{NH}_{2}$ was due to functionalisation with APTS that introduced some organic content on the nanoparticles surface. Also the LbL coating with the two different polymers resulted in similar amount of drug/polyelectrolytes deposited (Figure 2) and about 3-4\% each quadruple layer, moreover the thickness of the coating was negligible as seen in the TEM images (Figure 2); both results are in agreement with the general trend observed in other works ${ }^{34,38}$.

The zeta potential of the functionalised particles pre $\mathrm{LbL}(+20 \mathrm{mV})$ was due to the presence of the amino groups present in the APTS and similar to those reported previously ${ }^{16,38}$. The zig-zag pattern observed during the $\mathrm{LbL}$ is typical of the process ${ }^{41,42}$ and the lowest value (-30 mV) corresponds to the pure alginate in the same buffer $(\mathrm{pH}=5)$ while the values of $+8 \mathrm{mV}$ and $+4 \mathrm{mV}$ are those corresponding to the pure PBAE and gentamicin. The observation that after each deposited layer the zeta potential of the particle surface was equivalent to that of the pure coating molecule (polyelectrolyte or drug) indicated that the surface was fully covered and that the process parameters (concentration, $\mathrm{pH}$ and time) were correct. 


\subsection{Gentamicin release}

The highest concentration of gentamicin, observed after the first 24 hours in contact with the release buffers, was about $0.3 \mathrm{mg} / \mathrm{ml}$ for all samples; as the solubility of gentamicin is at least $10 \mathrm{mg} / \mathrm{ml}$ (as used in the LbL protocol), sink conditions were achieved (ratio between fluid concentration and maximum solubility was at $\sim 30$ and the minimum required is 3$).{ }^{43}$

The release from the LbL coated nanocarriers is a process governed by two distinct mechanisms, diffusion of the drug through the deposited layers and the delamination of the coating due to polyelectrolyte hydrolysis ${ }^{38,44}$. The hypothesis that led to the numerous applications of PBAEs in LbL for biomedical application is their ability to undergo hydrolysis and thus release the drug entrapped in the LbL coating ${ }^{44-47}$. Such view was recently confuted with evidences suggesting that drug diffusion through the deposited layers is in fact the controlling mechanism ${ }^{38}$. In this work, we used two different polymers compared to $\mathrm{Al}$ Thaher et al. ${ }^{38}$, even though still PBAEs, consequently, the positive fitting of the release profile with a first order kinetic model further reinstates that the controlling mechanism when PBAEs are employed in LbL is diffusion and not hydrolysis of the polyelectrolyte that would have determined a zero order kinetic of release (constant release throughout until all drug is released); furthermore no hydrolysis was detected for either PBAEs for at least 10 days (Figure 1). Antibiotic loading on the carriers was estimated as described by $\mathrm{Al}$ Thaher et al. ${ }^{38}$ and comparable to the results presented for nanoparticles coated with 5 quadruple layers.

In this work PMMA bone cement containing gentamicin powder $(4 \% \mathrm{w} / \mathrm{w})$ was used as control as this represents a commercial formulation of antibiotic laden bone cements. Despite the lower amount of gentamicin present in the bone cement samples when loaded onto the nanocarriers, as the loading of the gentamicin onto the nanoparticles was about $\sim 12 \%$ and the nanoparticles were added to concentration of $15 \% \mathrm{w} / \mathrm{w}$, the release profile of the antibiotic from PMMA bone cements containing the developed delivery systems was considerably longer (Figure 3b). It was also observed that the release from the commercial formulation did not follow a first order kinetic; hence confirming the questionable suitability of simply mixing antibiotics in PMMA bone cement if prolonged and controlled release is sought. Moreover, from a mass transfer phenomenon stand point, the release of 
gentamicin from the commercial bone cement depends only on the diffusion of the antibiotic in the PMMA matrix; on the other hand when the Si nanocarriers are added, as they distributed homogeneously in the PMMA bone cement matrix as seen in Figure S3, the overall gentamicin release is the sum of the diffusion first through the LbL coating and then through the PMMA matrix. The overall kinetic of diffusion through multiple phases is controlled by the slowest of the diffusion kinetics. Therefore, if diffusion through the LbL coating was "faster" than diffusion through PMMA bone cement, the addition of Si nanocarriers would result in the same release profile between commercial formulation and bone cement with the drug delivery system as, regardless of the ability of the antibiotic to migrate through the LbL coating, the overall diffusion would be controlled by the diffusion through the PMMA phase. On the contrary, if the diffusion through the LbL coating was "slower", the release profile between commercial formulation would exhibit a quick release kinetic than the samples containing the Si nanocarriers; moreover bone cement containing the nanocarriers would also would show a release profile similar to that of the Si nanocarriers simply suspended in solution as only the antibiotic that is continuously released from the nanocarriers can be transported through the PMMA bone cement matrix. Our results (Figure 3a and b) demonstrate that the release from the nanocarriers is the controlling step in the overall antibiotic release process from bone cement. The prolonged released from the bone cement resulting from the use of the coated silica nanocarriers is due to gentamicin becoming available after diffusing through the LbL coating as time progresses while in commercial formulation all the antibiotic is available from the beginning. Of the total antibiotic initially contained in the PMMA bone cement samples, more was released when silica nanocarriers were used (>-50\%) while when gentamicin powder was simply mixed only $15 \%$ was released (Figure 3b). The later observation is consistent with literature ${ }^{48,49}$ and the general explanation is entrapment inside the PMMA matrix preventing the antibiotic migration from the PMMA bone cement bulk to the surface. However, the greater amount of antibiotic capable of leaving the PMMA bone cement when the silica nanocarriers were used could also be the result of the protection of the drug from the potentially damaging radical species that drive the polymerisation reaction during bone cement curing. This protective action would not be available to the drug molecules when simply mixed as in the commercial formulation. Hence, the known fact that only 
about a fifth of antibiotic contained in the PMMA bone cement is released would not be due to entrapment but to drug damage during bone cement curing. It is well known that gentamicin, like tobramycin, can survive high temperatures (even autoclave) and this is one of the reasons for their use in bone cements where the radical polymerisation reaction can result in temperatures as high as 60-70 ${ }^{\circ} \mathrm{C}$,; however the ability to withstand high temperature does not necessarily mean resistance to radicals. This protective hypothesis would also explain the similar result observed when gentamicin was introduced in PMMA bone cement through liposome ${ }^{37}$.

\subsection{Antimicrobial activity}

Despite the remarkable prolonged release of gentamicin when the nanocarriers were employed compared to the commercial formulation where the drug is mixed, it was critical to assess the ability to prevent bacterial growth as the released drug could not provide the required effect in virtue of the nanocarriers preparation and subsequent release; similarly bone cement nanocarriers prepared using only alginate and POLY1 or POLY2 were tested to identify any possible antimicrobial activity due to the polyelectrolytes.

The antimicrobial test was especially designed to evaluate the length of the antimicrobial period exhibited by the PMMA bone cement containing the drug delivery system and the bacterial species tested represent the typical pathogens found in PJI ${ }^{50-52}$.

The principle is based on the fact that bacteria are able to grow in any media when the concentration of antibiotic is below a certain value (MIC) that depends on the specific bacteria strain. The concentration of gentamicin in the release buffer decrease with time; hence the microorganisms will only be able to growth in release media collected after a certain time (as the release buffer was changed daily). The absence of antimicrobial activity from the control sample was a confirmation of the cytocompatibility of the polyelectrolytes employed in the LbL assembly process. The susceptibility to the antibiotic released varied among the bacterial species with clinical isolates generally more resistant, and it was generally in line with the MIC of gentamicin for the different species (supplementary info); furthermore, this observation reinforces the notion that technology are better tested using clinical isolates than catalogue strains. PMMA bone cement with mixed gentamicin 
powder antimicrobial was capable of preventing growth of many of the bacteria species for about 1 week, in line with the general expectation; while very weak (low MIC) were inhibited for longer. For example, the longest activity was observed against the weakest bacteria (S. epidermidis ATCC12228) and terminated when the release terminated. The use of the silica nanocarriers resulted in all the bacteria species growth being inhibited by the released antibiotic for longer than in case gentamicin powder in agreement to higher the concentrations of antibiotic in the release buffer for longer periods of time (Figure 3). Additionally, the PMMA bone cement containing the silica nanocarriers prepared using POLY2 for shorter period of time than those prepared with POLY1 was consistent with the concentration in the release buffer in contact with POLY2 samples exhibiting lower antibiotic concentrations than those with POLY1.

\subsection{Bone cement properties}

The material properties of PMMA bone cement are satisfactory therefore the addition of the silica nanocarriers did not have the objective to improve any specific parameter; however, it was important to verify that the encapsulation of the developed silica nanocarriers did not have negative impact on the PMMA bone cement properties. The mechanical properties (water uptake, compression, bending and fracture resistance) did not improve despite the use of nanoparticles that resulted in a nanocomposite material; this is likely because the PMMA bone cement contains already a considerable amount of inorganic particulate $\left(\mathrm{BaSO}_{4}\right)$ thus the addition of nanoparticles had a negligible impact. Water uptake is due to swelling; when the nanocarriers are used the concentration of the polymeric matrix and $\mathrm{BaSO}_{4}$ decreased but the nanocarriers are also capable of water uptake compensating for the reduction of these components.

Rheological properties are important for surgeons as they need enough time to apply the bone cement but curing needs to be quick enough to allow speedy patient mobilisation and rehabilitation; current surgical techniques require the bone cement to be mixed for about 1-1.5 min and applied in less than $\sim 5$ min from initial mixing. The fact that silica nanocarrier do not alter the curing properties of PMMA bone cement would allow orthopaedic surgeons to perform joint replacement operation 
without having to change their technique so retraining would not be required hence reducing time and cost associated to switching to the novel bone cement.

Another important assessment of the novel PMMA bone cement was the response of osteoblast cells when in contact with such material compared to the commercial formulation. No only cell viability but also other aspect of osteoblast metabolism such wall morphology and calcium production was assessed and no detrimental impact of the nanocarriers observed in any of these. Particularly calcium production is linked to the ability of the cells to integrate the abiotic surface into the bone while the alteration of the cell morphology could indicate inflation with the possible alteration of the balance between osteoblasts and osteoclasts toward the former cell type with consequent bone resorption overtaking bone formation; a process well known to result in aseptic loosening and joint replacement failure.

\subsection{Conclusions}

PMMA bone cement containing antibiotic is the gold standard material used during joint replacement device implantation to fix the device in place and to provide a source of antimicrobial agent to prevent infection offset after surgery. Despite the wide diffusion of this material, infections still pose a major threat to patient undergoing this type of orthopaedic procedures; predominantly because the antibiotic is fast released from the bone cement while infection can establish well after surgery even months. To overcome this limitation, we have developed novel antibiotic nanocarriers depositing the drug on the surface of silica nanoparticles using the Layer-by-Layer technique and hydrolysable and inexpensive polyelectrolytes (poly beta amino esters).

When the antibiotic was added to PMMA bone cement using these new drug delivery systems, drug release and the antimicrobial activity were protracted to almost 1 month compared to $\sim 1$ week the commercial formulation. The prolong antimicrobial activity was not achieved compromising any of the multitudes of bone cement properties tested hence the newly developed nanocarriers can provide a viable solution to the fight against late prosthetic joint infection that pose a serious rick to patient quality of life with potentially lethal consequences and constitute a significant economic burden to the financial resources of the health care providers. 


\section{Acknowledgements}

This work was supported by the Life Science Research Network Wales, an initiative funded through the Welsh Government's Sêr Cymru program (project 507444).

The authors would also like to thank Prof. Alasdair MacGowan (Public Health England, North Bristol NHS Trust \& University of Bristol) and Dr. Karen Bowker (North Bristol NHS Trust) for providing clinical isolates of PJI.

Part of the work was performed under the umbrella of the European COST action MP1106 "Smart and Green Interfaces, from single bubbles and drops to industrial, environmental, and biomedical applications".

\section{Supporting information}

Generalised linear models, fitting results (coefficients, plots, residues), gentamicin MIC of the bacterial species employed.

\section{References}

1 National Joint Registry. 2017. "National Joint Registry.” NJR StatsOnline http://wwwnew.njrcentre.org.uk/njrcentre/Healthcareproviders/Accessingthedata/StatsOnline/NJRStatsOnlin e/tabid/179/Default.aspx

2 Hansen E.N., Adeli B., Kenyon R., Parvizi J. Routine Use of antibiotic Laden bone Cement for Primary Total Knee Arthroplasty: Impact on Infecting Microbial Patterns and Resistance Profiles. J. Arthroplasty. 2014;29(6):1123-1127

3 Tsaras G, Osmon DR, Mabry T, Lahr B, St Sauveur J, Yawn B, Kurland R, Berbari EF. Incidence, Secular Trends, and Outcomes of Prosthetic Joint Infection: a Population-Based Study, Olmsted county, Minnesota, 1969-2007. Infect. Control Hosp. Epidemiol. 2012;33:12071212.

4 Anagnostakos K, Fürst O, Kelm J. Antibiotic-impregnated PMMA Hip Spacers: Current Status. Acta Orthop. 2006;77:628-37 
5 Anagnostakos K., Schmid N.V., Kelm J., Grün U., Jung J. Classification of Hip Joint Infections. Int. J. Med. Sci. 2009; 6(5): 227-233.

6 Vanhegan IS, Malik AK, Jayakumar P, Ul Islam S, Haddad FS. A Financial Analysis of Revision Hip Arthroplasty: the Economic Burden in Relation to the National Tariff. J. Bone Joint Surg. Br.2012; 94:619-623.

7 Kurtz SM, Lau E, Watson H, Schmier JK, Parvizi J. Economic Burden of Periprosthetic Joint Infection in the United States. J. Arthroplasty 2012;27:61-65.e61.

8 Sendi P., Banderet F., Graber P., Zimmerli W. Clinical Comparison Between Exogenous and Haematogenous Periprosthetic Joint Infections Caused by Staphylococcus aureus. Clin. Microbiol. Infect. 2011;17:1098-1100

9 Zimmerli W, Trampuz A, Ochsner PE. Prosthetic-joint Infections. N. Engl. J. Med. 2004; 351: $1645-1654$

10 Tande A.J., Patel R. Prosthetic Joint Infection. Clinical Microbiol. Rev. 2014:27(2):302-345

11 Al-Thaher Y., Perni S., Prokopovich P. Nano-carrier Based Drug Delivery Systems for Sustained Antimicrobial Agent Release from Orthopaedic Cementous Material. Adv. Coll. Interface Sci. 2017;249:234-247

12 Hickok N.J., Shapiro I.M. Immobilized Antibiotics to Prevent Orthopedic Implant Infections. Adv Drug Deliv Rev. 2012;64(12):1165-1176.

13 Monteiro N., Martins M., Martins A., Fonseca N.A., Moreira J.N., Reis R.L., Neves N.M. Antibacterial Activity of Chitosan Nanofiber Meshes with Liposomes Immobilized Releasing Gentamicin. Acta Biomater. 2015;18:196-205

14 Goissis G., Sousa M.H. Characterization and in vitro Release Studies of Tetracycline and Rolitetracycline Immobilized On Anionic Collagen Membranes. Mat. Res. 2009;12(1):69-74

15 Ahangari A., Salouti M., Heidari Z., Kazemizadeh A.R., Safari A.A. Development of Gentamicin-Gold Nanospheres for Antimicrobial Drug Delivery to Staphylococcal infected foci. Drug Deliv. 2013;20(1):34-39

16 Perni S., P. Prokopovich P. Continuous Release of Gentamicin from Gold Nanocarriers. RSC Advances 2014;4:51904-51910 
17 Motamedi H., Mazdeh S.K., Azim A.K., Mehrabi M.R. Optimization of Gold Nanoparticle Biosynthesis by Escherichia coli DH5[alpha] and its Conjugation with Gentamicin. J. Nano Res. 2015;32:93-105

18 Salouti M., Heidari Z., Ahangari A., Zare S. Enhanced Delivery of Gentamicin to Infection Foci Due to Staphylococcus aureus Using Gold Nanorods. Drug Deliv. 2016;23(1):49-54

19 Capeletti L.B., de Oliveira L.F., Gonçalves K. de A., de Oliveira J.F., Saito Â., Kobarg J., dos Santos J.H., Cardoso M.B. Tailored silica-Antibiotic Nanoparticles: Overcoming Bacterial Resistance with Low Cytotoxicity. Langmuir. 2014;30(25):7456-7464

20 Mugabe C., Azghani A.O., Omri A. Liposome-mediated Gentamicin Delivery: Development and Activity Against Resistant Strains of Pseudomonas Aeruginosa Isolated from Cystic Fibrosis Patients. J. Antimicrob. Chemother. 2005;55(2):269-671

21 Chuang H.F., Smith R.C., Hammond P. T. Polyelectrolyte Multilayers for Tunable Release of Antibiotics. Biomacromolecules 2008;9:1660-1668.

22 Moskowitz J.S., Blaisse M.R., Samuel R.E., Hsu H.P., Harris M.B., Martin S.D., Lee J.C., Spector M., Hammond P.T. The Effectiveness of the Controlled Release of Gentamicin from Polyelectrolyte Multilayers in the Treatment of Staphylococcus aureus Infection in a Rabbit Bone Model. Biomaterials. 2010;31(23):6019-6030

23 Tamanna T, Bulitta JB, Yu A. Controlling Antibiotic Release from Mesoporous Silica Nano Drug Carriers via Self-Assembled Polyelectrolyte Coating. J. Mater. Sci. Mater. Med. 2015;26:17.

24 Shen S.C., Ng W.K., Dong Y.C., Ng J., Tan R.B.H. Nanostructured Material Formulated Acrylic Bone Cements with Enhanced Drug Release. Mater. Sci. Eng. C 2016;58:233-241

25 Ayre W.N., Birchall J.C., Evans S.L., Denyer S.P. A Novel Liposomal Drug Delivery System for PMMA Bone Cements. J. Biomed. Mater. Res. B Appl. Biomater. 2015:104:1510-1524

26 Lu Z., Eadula S., Zheng Z., Xu K., Grozdits G., Lvov Y. Layer-by-layer Nanoparticle Coatings on Lignocellulose Wood Microfibers. Colloids and Surfaces A: Physicochemical and Engineering Aspects 2007; 292 (1):56-62 
27 Shah N.J., Hong J., Hyder M.N., Hammond P.T. 2012. Osteophilic Multilayer Coatings for Accelerated Bone Tissue Growth. Adv. Mater. 24(11), 1445-1450.

28 Kovačević D., Pratnekar R.,, Torkar K.G., Salopek J., Dražić G., Abram A., Bohinc K. Influence of Polyelectrolyte Multilayer Properties on Bacterial Adhesion Capacity. Polymers 2016;8(10):345

29 Al Alotaibi H.F., Perni S., Al Thaher Y, Prokopovich P. Role of Processing Parameters on Surface and Wetting Properties Controlling the Behaviour of Layer-by-Layer Coated Nanoparticles. Cur. Opinion Coll. Interface Sci. 2018;249:234-247

30 Ngamcherdtrakul W., Morry J., Gu S., Castro D.J., Goodyear S.M., Sangvanich T., Reda M.M., Lee R., Mihelic S.A., Beckman B.L., Hu Z., Gray J.W., Yantasee W. Cationic Polymer Modified Mesoporous Silica Nanoparticles for Targeted SiRNA Delivery to HER2+ Breast Cancer. Adv. Funct. Mater. 2015;25(18):2646-2659

31 He Q.J., Shi J.L. Mesoporous Silica Nanoparticle Based Nano Drug Delivery Systems: Synthesis, Controlled Drug Release and Delivery, Pharmacokinetics and Biocompatibility. J. Mater. Chem. 2011;21(16):5845-5855

32 Lee C. The Well-Cemented Total Hip Arthroplasty; Springer Berlin Heidelberg, 2005; pp. 6066.

33 Yildirim A., Ozgur E., Bayindir M. Impact of Mesoporous Silica Nanoparticle Surface Functionality on Hemolytic Activity, Thrombogenicity and Non-Specific Protein Adsorption. J. Mater. Chem. B 2013;1(14):1909-1920

34 Perni S., Martini-Gilching K., Prokopovich P. Controlling Release Kinetics of Gentamicin from Silica Nano-Carriers. Coll. Surfaces A 2018;541:212-221

35 Tangy F., Moukkadem M., Vindimian E., Capmau M.L., Le Goffic F. Mechanism of Action of Gentamicin Components. Characteristics of Their Binding to Escherichia coli Ribosomes. Eur. J. Biochem. 1985;147(2):381-386

36 Mascaretti O.A. Bacteria Versus Antimicrobial Agents - An Integrated Approach. American Society Microbiology Press 2003 
37 Hinarejos P., Guirro P., Puig-Verdie L., Torres-Claramunt R., Leal-Blanquet J., Sanchez-Soler J., Carles Monllau J. Use of Antibiotic-loaded Cement in Total Knee Arthroplasty. World J. Orthop. 2015;6(11):877-885

38 Al-Thaher Y., Latanza S., Perni S., Prokopovich P. Role of Poly-beta-amino-esters Hydrolysis and Electrostatic Attraction in Gentamicin Release from Layer-by-Layer Coatings. J. Coll. Interface Sci. 2018;:526:35-42

39 Perni S., Yang L., Preedy E.C., Prokopovich P. Cobalt and Titanium nanoparticles influence on human osteoblast mitochondrial activity and biophysical properties of their cytoskeleton. J. Coll. Interface Sci. 2018;531:410-420.

40 Bates D, Maechler M, Bolker B., Walker S.. Fitting Linear Mixed-Effects Models Using lme4. J. Statistical Software 2015;67(1):1-48.

41 Cristofolini L., Szczepanowicz K., Orsi D., Rimoldi T., Albertini F., Warszynski P. Hybrid Polyelectrolyte/Fe3O4 Nanocapsules for Hyperthermia Applications. ACS Appl. Mater. Interfaces. 2016;8(38):25043-25050

42 Szczepanowicz K., Dronka-Góra D., Para G., Warszyński P. Encapsulation of Liquid Cores by layer-by-layer Adsorption of Polyelectrolytes. J. Microencapsul. 2010;27(3):198-204

43 The United States Pharmacopeia: The National Formulary (USP37/NF32). The United States Pharmacopeial Convention, Inc; Rockville, MD: 2014

44 Smith, R.C., Riollano M., Leung A., Hammond P.T. 2009. Layer-by-Layer Platform Technology for Small-Molecule Delivery. Angewandte Chemie International Edition 48(47), 8974-8977.

45 Chuang, H.F., Smith R.C., Hammond P.T. 2008. Polyelectrolyte Multilayers for Tunable Release of Antibiotics. Biomacromolecules 9(6), 1660-1668

46 Min J., Choi K.Y., Dreaden E.C., Padera R.F., Braatz R.D., Spector M., Hammond P.T. 2016. Designer Dual Therapy Nanolayered Implant Coatings Eradicate Biofilms and Accelerate Bone Tissue Repair. ACS Nano. 10(4), 4441-4450

47 Moojen, D.J.F., Hentenaar B., Charles Vogely H., Verbout A.J., Castelein R.M., Dhert W.J. 2008. In vitro Release of Antibiotics from Commercial PMMA Beads and Articulating Hip Spacers. The Journal of Arthroplasty 23(8), 1152-1156. 
48 Wu K., Chen Y.C., Hsu Y.M., Chang C.H. Enhancing Drug Release from Antibiotic-loaded Bone Cement Using Porogens. J. Am. Acad. Orthop. Surg. 2016;24:188-195

49 Wendling A., Mar D., Wischmeier N., Anderson D., McIff T. Combination of Modified Mixing Technique and Low Frequency Ultrasound to Control the Elution Profile of Vancomycin-Loaded Acrylic Bone Cement. Bone Joint Res. 2016;5:26-32

50 Geipel U. Pathogenic Organisms in Hip Joint Infections. Inter. J. Med. Sci. 2009;6(5):234-240

51 Martínez-Pastor J.C., Mahamud E.M., Vilchez F., García-Ramiro S., Bori G., Sierra J., Martínez J.A., Font L., Mensa J., Soriano A. Outcome of Acute Prosthetic Joint Infections Due to GramNegative Bacilli Treated with Open Debridement and Retention of the Prosthesis. Antimicrobial Agents Chemother. 2009;53(11):4772-4777

52 Corvec S., Illiaquer M., Touchais S., Boutoille D., van der Mee-Marquet N., Quentin R., Reynaud A., Lepelletier D., Bémer P. Bone and Joint Infection Study Group. Clinical features of group B Streptococcus Prosthetic Joint Infections and Molecular Characterization of Isolates. J. Clin. Microbiol. 2011;49(1):380-382 
